\title{
ORDENACIÓN Y PLANIFICACIÓN REGIONAL DEL SUELO INDUSTRIAL. ASTURIAS Y SU ENTORNO REGIONAL ${ }^{1 *}$
}

\author{
P. Benito del Pozo \\ Departamento de Geografía y Geología \\ Universidad de León \\ mpbenp@unileon.es
}

\begin{abstract}
Resumen: La importancia del suelo industrial en la consolidación de los tejidos productivos explica el interés de las Administraciones Públicas por regular su ordenación y planificación, no desde el punto de vista urbanístico, competencia de los ayuntamientos, sino como infraestructura económica y territorial ligada a la política regional. Especial interés reviste el tratamiento de este uso del suelo en las directrices regionales de ordenación del territorio y en los planes de suelo impulsados por las Comunidades Autónomas. La experiencia planificadora de los gobiernos regionales en el norte peninsular, en particular en Asturias, ilustra el alcance y significado de este fenómeno y sus implicaciones territoriales, pues la localización industrial refuerza los procesos de urbanización, el dinamismo de las áreas metropolitanas, de las periferias urbanas y de ciertos espacios rurales, al tiempo que los agentes económicos son más exigentes con la calidad de los asentamientos industriales, lo que favorece promociones mejor dotadas y menos especulativas.
\end{abstract}

Palabras clave: planificación regional, ordenación de espacios industriales, política de suelo industrial, desarrollo territorial.

\begin{abstract}
The importance of industrial land in the consolidation of productive explains the growing interest of government to regulate their management and planning. The treatment that this type of soil receives in the regional guidelines for spatial and territorial planning driven by the Autonomous Communities is of particular interest. Planner's experi-
\end{abstract}

\footnotetext{
${ }^{1}$ Este trabajo se inscribe en el Proyecto Ordenación, planificación y gestión de las áreas empresariales en el norte de España. Ministerio de Ciencia e Innovación, 2010. Referencia: CSO2010-18471.

* Recibido: 18-10-10. Aceptado: 23-06-11.
} 
ence in northern Spain, particularly in Asturias, serves to illustrate the scope and significance of this phenomenon, its geographic significance and its implications for territorial development, since industrial location reinforces the urbanization processes and favours dynamism of the metropolitan areas, the urban peripheries and some rural areas, while operators are becoming more demanding with the quality of industrial settlements, which favours better equipped and less speculative promotions.

Keywords: regional planing, management of industrial areas, industrial land policy, territorial development.

\section{Introducción}

El interés social por el territorio y su desarrollo ordenado ha impulsado, desde principios de los años de 1990 en España, la puesta en marcha de un repertorio cada vez más amplio y complejo de instrumentos que intentan regular los usos del suelo, ya no sólo los residenciales, piedra angular del urbanismo, sino también los económicos. Las ciudades y los núcleos urbanos crecen a impulsos de un variado repertorio de actividades que sustentan el crecimiento de la población. Esas actividades productivas (industrias y servicios) son voraces consumidoras de suelo y, por ello, un factor de urbanización de primer orden. No se puede construir ni proyectar la ciudad y su entorno sin considerar estos usos productivos. Tampoco las políticas de desarrollo regional pueden obviar los procesos económicos que inciden en la articulación del territorio y favorecen el progreso, debiendo incorporar la sostenibilidad como requisito insoslayable. Tales premisas se siguen de la necesaria intervención de los poderes públicos para ordenar y planificar la dimensión, localización y distribución de los espacios económicos en el territorio (BIELZA, 2008; ROMERO y FARINÓS, 2004).

Este trabajo plantea una revisión de los criterios y el alcance de la experiencia planificadora del suelo industrial en el norte de España, con especial atención al caso de Asturias, donde los instrumentos están bien definidos y desarrollados desde fecha temprana, siendo que la región fue pionera en aprobar unas directrices de ordenación del territorio y también una de las primeras en planificar el suelo industrial desde entidades oficiales de rango autonómico con un doble enfoque sectorial/territorial.

La metodología se basa en el análisis de los principales documentos de ordenación y planificación, el rastreo de publicaciones y fuentes oficiales y el trabajo de campo, para cubrir lagunas informativas y actualizar datos. Se trata de demostrar la 
hipótesis de que la planificación del suelo industrial es asumida como una necesidad por los poderes públicos, en razón de tres factores que influyen en la toma de decisiones: uno, por la presión de la demanda (los empresarios reclaman suelo industrial en cantidad y a precios competitivos, en un mercado del que están prácticamente ausentes los promotores privados); dos, por la importancia de la actividad industrial -y empresarial en general- como motor de la economía regional; y tres, por el compromiso político con la ordenación del territorio y el consumo sostenible de suelo.

\section{Contexto normativo de los programas de suelo industrial}

El marco jurídico en el que se desenvuelven los programas de promoción de suelo industrial en España entraña una notable complejidad debido a la abundante legislación concurrente (comunitaria, estatal y autonómica), la diversidad de sus fuentes y la superposición de competencias de las Administraciones Públicas que intervienen para generar nuevo suelo destinado a usos industriales y empresariales. Los programas o planes de suelo industrial se enmarcan, por lo general, en las políticas industriales y deben ser coherentes con las demás políticas sectoriales (medio ambiente, infraestructuras de transporte, agua y energía, etcétera) y con las políticas territoriales (ordenación del territorio y urbanismo). Además, en la programación de suelo industrial hay que tener en cuenta la regulación existente en la ayuda pública a las empresas (con límites a la misma) y en los mecanismos de financiación y comercialización del suelo para actividades productivas (SANZ, 1982; PUJADAS y FONT, 1998).

En lo que sigue se revisan de forma sintética los aspectos de la legislación europea, nacional y regional que afectan a los programas de suelo industrial, haciendo hincapié en los criterios e instrumentos de ejecución y financiación de los planes oficiales de suelo industrial.

En España la ordenación y planificación del suelo industrial es competencia de los Gobiernos autonómicos. Las propuestas y actuaciones en tal materia deben estar en consonancia, ya se ha dicho, con las políticas sectoriales y territoriales, de manera que unas y otras sean coherentes entre sí. Por su parte y a este respecto, la Unión Europea establece, tanto en política industrial como territorial, directrices o recomendaciones, al tiempo que elabora programas que incentivan la aplicación de dichas estrategias en los Estados miembro y fija los mecanismos de control y evaluación de los mismos (COMISIÓN EUROPEA, 1999 y 2007). Es decir, hay una intervención y una regularización clara, aunque su eficacia depende de la interpretación concreta que cada Estado hace de la misma, lo que abre fisuras que pueden derivar en fricciones territoriales. De hecho, es muy difícil armonizar las medidas y actuaciones concretas a escala de país y región. 
Las regiones o comunidades autónomas que en España se proponen desarrollar programas de promoción de suelo industrial deben adecuar los instrumentos financieros a las necesidades del período para el que se contempla la actuación (un mínimo de cuatro años), a fin de conseguir una mayor eficacia en la gestión de los fondos disponibles, favoreciendo la modernización y diversificación del tejido empresarial, así como la creación de empleo estable. Además de los ajustes instrumentales, para la promoción de espacios industriales se debe atender a las disposiciones emitidas por el legislador comunitario en los siguientes aspectos:

a) Las ayudas a las empresas y los elementos que se consideran ayudas en las ventas de terrenos y construcciones por parte de los poderes públicos.

b) La Evaluación Estratégica Ambiental y la Evaluación Conjunta de Impacto Ambiental, ya que los programas de suelo industrial pueden implicar cambios en la clasificación o calificación del suelo y, por lo tanto, exigir la tramitación del correspondiente instrumento ambiental.

Sobre el primer aspecto, la disposición de referencia es la Comunicación 97/ C 209/03 de la Comisión, que establece las Directrices destinadas a los Estados Miembros en las que se describe el procedimiento mediante el cual la venta de suelo y edificación por parte del Estado no se considera ayuda estatal y establece en qué casos esas ventas tienen que ser notificadas a la Comisión. El otro aspecto a tener en cuenta es el medioambiental, para lo que se acude a las Directivas comunitarias 85/337/CE, de 28 de junio y 97/11/CE, de 3 de marzo, las cuales regulan la Evaluación de Impacto Ambiental (EIA) como procedimiento y técnica de evaluación de las alteraciones ambientales en proyectos públicos o privados que se ejecutan sobre el medio. Un complemento de la misma es la llamada Evaluación Ambiental Estratégica, alusiva a todos los niveles de toma de decisiones que anteceden al proyecto. Estas Directivas ya han sido traspuestas a la legislación española mediante la Ley 9/2006 y a las correspondientes legislaciones autonómicas.

El marco legal estatal sobre promoción de suelo industrial está definido por el derecho general en materia de ordenación del territorio y urbanismo y por la normativa autonómica en dicha materia. De ineludible mención es la Ley de Suelo 8/2007, de 28 de mayo, de especial incidencia en la gestión del suelo industrial, pues establece una nueva regulación de las valoraciones del suelo a efectos de reparcelación y expropiación. En efecto, el anterior sistema valoraba los terrenos teniendo en cuenta su clasificación futura, esto es, partiendo de cuál fuera su destino final y no su situación real, por lo que se incorporaban en la valoración las expectativas venideras; con el nuevo sistema vigente se valoran los suelos en función de su calificación actual, sin tener en cuenta la situación futura, lo que supone en la práctica un menor coste de adquisición. 
Otro aspecto a resaltar es la regulación estatal sobre evaluación ambiental asociada a la tramitación urbanística de nuevos desarrollos de suelo o a la implantación de empresas industriales. Destaca el Real Decreto Legislativo 1.302/1986 que regula el procedimiento de Evaluación de Impacto Ambiental (EIA) y determina los proyectos de instalaciones industriales que necesitan una EIA para la concesión de licencias. A su vez, la Ley 2/2006, sobre evaluación de los efectos de determinados planes y programas en el medio ambiente, establece los planes y programas de ordenación del territorio en los ámbitos urbano y rural que serán objeto de Evaluación Ambiental Estratégica (EAE). Se establece así en qué casos la implantación de un asentamiento industrial necesita la tramitación, junto con el documento urbanístico pertinente (plan parcial), del documento de Evaluación Ambiental Estratégica, y, a su vez, fija qué proyectos de las empresas que se instalen necesitarán una evaluación de impacto ambiental.

En definitiva, este entramado legal se traduce, con frecuencia, en una tramitación lenta y cargada de burocracia que impide a los responsables de la política industrial disponer del suelo empresarial necesario para atender con agilidad a las demandas espontáneas y que pone freno al desarrollo territorial, pues se pueden perder oportunidades de inversión y refuerzo del tejido empresarial. Por ende, también este factor aviva la competencia territorial: las comunidades autónomas rivalizan por ofertar suelo empresarial de ágil gestión y condiciones ventajosas.

\section{La regulación del suelo y los espacios industriales: el ejemplo de Asturias}

En España la situación del suelo industrial varía de unas regiones a otras, pero hay elementos comunes muy similares entre sí (MÉNDEZ, 2006). El caso de Asturias ilustra bien la complejidad del entramado legal antes referido, a la vez que permite extrapolar elementos y situaciones reguladoras, pues comparte los planteamientos de base con el resto de las comunidades autónomas, en particular con Galicia, Cantabria y Castilla y León. En unas y otras existen directrices de ordenación del territorio, planes sectoriales/territoriales y una legislación urbanística que, aunque con ritmos de tramitación distintos, traslucen el afán de los responsables públicos por poner límite al crecimiento descontrolado del suelo industrial, regular directa o indirectamente su desarrollo y propiciar, en última instancia, el desenvolvimiento sostenible de la actividad fabril (ROMERO y FARINÓS, 2004).

Por su evidente dimensión territorial y por ser el instrumento normativo más expresivo de los criterios sobre suelo industrial, es oportuno detenerse, en primer término, en las disposiciones de las Directrices Regionales de Ordenación del Territorio, 
aprobadas por el Principado de Asturias en enero de 1991 (CONSEJERÍA DE MEDIO AMBIENTE Y URBANISMO, 1992). En este documento destacan tres directrices que hacen mención al suelo para actividades económicas. La Directriz $3^{\underline{a}}$ determina los tipos de actuaciones sujetas a evaluación de impacto estructural, entre las que se incluyen las áreas industriales del tipo "Gran Industria" y "Grandes polígonos industriales". Ambas están sujetas a evaluación de impacto estructural al ser necesario valorar, por un lado, los costes que conlleva su implantación y, por otro, los beneficios económicos y sociales que se derivan directamente de dicha actuación (BENITO, 2006).

La Directriz $7^{\text {a }}$ establece los criterios para la distribución y tratamiento de áreas industriales, a saber: a) debe evitarse la dispersión excesiva del suelo industrial, pues el crecimiento discontinuo del mismo encarece los costes de los factores relacionados con la accesibilidad, urbanización y servicios necesarios. Este criterio de concentrar las actuaciones de suelo para actividades económicas se encuentra a su vez recogido en la Ley de ordenación territorial y urbanística de Asturias; b) el dimensionamiento del suelo industrial debe ser realista en cuanto a la previsión de implantación de nuevas industrias y ampliación de las existentes. Se recomienda observar, en lo posible, que las cabeceras de comarca y subcomarca funcional se doten de un mínimo suelo industrial en polígono, si el medio y otras circunstancias lo permiten; c) deben evitarse inversiones en suelo que quede sin utilizar largos períodos de tiempo; y d) en el diseño de las zonas industriales deberá preverse el espacio y normativa necesarios para las actividades de servicios ligadas a la producción industrial, de manera que la separación entre ambas no se vea forzada por el planeamiento urbanístico.

En consecuencia, la promoción pública de suelo industrial en Asturias plantea respetar, en primera instancia, el principio de reequilibrio territorial en lo que respecta a la localización y distribución de los asentamientos industriales; para ello se aplican criterios de supramunicipalidad. En segundo lugar, propone atender las necesidades de los espacios periféricos con una oferta especializada y adecuada a sus potencialidades. En tercer lugar, pretende potenciar las economías de escala, sobre todo en las zonas con mejores equipamientos e infraestructuras, como es el caso de la Zona Central, donde se localizan las ciudades que articulan el Área Central (Oviedo, Gijón, Avilés, Mieres y Langreo).

Asimismo, la Directriz 8 establece como recomendación la conveniencia de que el planeamiento coadyuve a potenciar las actividades industriales y terciarias en torno a Mieres y el eje urbano del Nalón, una indicación que intenta atajar el declive y la recesión en la cuenca central hullera. No obstante lo señalado, algunas condiciones descritas pueden variar en el futuro ya que las DROT están en proceso de revisión (el documento de Avance aparece publicado en el BOPA, 21 de marzo de 2006). 
Por su parte, la legislación urbanística asturiana regula aspectos específicos del suelo industrial, al igual que otras leyes autonómicas sobre la materia. El Decreto Legislativo 2/2004 establece, entre otros, los siguientes aspectos relativos al urbanismo industrial:

- El plan general de urbanismo calificará los usos industriales evitando la dispersión de este tipo de suelo.

- La figura de Espacios de Gestión Integrada se aplicará a los proyectos de suelo industrial que abarquen a más de un municipio.

- Se fijará una protección para el suelo ya calificado como industrial frente a la recalificación a otros usos, como el residencial.

- Los planes generales podrán incluir reservas de suelo que permitan el mantenimiento o la ampliación de actividades industriales en la franja de quinientos metros desde la ribera del mar, y que sean de difícil o imposible ubicación o traslado a otros lugares, siempre que se trate de industrias de interés regional.

- La figura de Reserva Industrial de Interés Regional permite clasificar como urbanizable el suelo anexo a una industria de interés regional.

- La implantación del uso industrial en suelo no urbanizable tiene carácter restrictivo. El plan general posibilitará su emplazamiento en pequeñas áreas industriales integradas en su entorno.

- Es obligatorio realizar cesiones de suelo para que los polígonos industriales cuenten con dotaciones que les hagan atractivos para las empresas.

- Se permite la autorización anticipada de usos industriales en las zonas en que el planeamiento autorice expresamente dichos usos.

\section{El desarrollo de suelo industrial: promotores e instrumentos}

Las directrices regionales de ordenación del territorio son vinculantes para todas las escalas de intervención (nacional, regional, municipal). En consecuencia, las políticas industriales y los planes de suelo industrial deben atender sus prescripciones y someterse a ellas, con lo que se pretende garantizar una programación ordenada del suelo para las empresas y un desarrollo del mismo según el modelo territorial asumido por cada Administración autonómica. En Asturias las Directrices son una realidad desde 1991 mientras que Castilla y León tardó en disponer de este instrumento (ver cuadro 1), lo que impidió durante casi dos décadas regular la ordenación de los espacios económicos e industriales con un criterio territorial preciso para las nueve provincias del espacio regional. En los casos de Galicia y Cantabria, a falta de la apro- 
bación definitiva de sus respectivos planes de ordenación territorial, las leyes de urbanismo cumplen dicha función (cuadro 1). Pero, ciertamente, quedan aspectos al descubierto, lo que abre una cierta brecha entre estas regiones y sus vecinas por ausencia de perspectiva territorial en la regulación de los usos empresariales del suelo (GAGO y GARCÍA, 1997).

Cuadro 1. Normativa en Asturias y comunidades limítrofes con prescripciones sobre suelo industrial

\begin{tabular}{|c|c|}
\hline CC.AA. & Tipo de documento y año de aprobación \\
\hline Asturias & $\begin{array}{l}\text { - Decreto Legislativo } 1 / 2004 \text {, de } 24 \text { de abril. Texto Refundido en materia } \\
\text { de ordenación del territorio y urbanismo. } \\
\text { - Directrices Regionales de Ordenación del Territorio de Asturias, } 1991 .\end{array}$ \\
\hline Galicia & $\begin{array}{l}\text { - Ley 15/2004, de } 29 \text { de diciembre, de Ordenación Urbanística y Medio } \\
\text { Rural de Galicia. } \\
\text { - Plan de Organización Territorial de la Xunta de Galicia (en tramitación). }\end{array}$ \\
\hline Cantabria & $\begin{array}{l}\text { - Ley } 2 / 2001 \text {, de } 25 \text { de junio, de Ordenación Territorial y Régimen } \\
\text { Urbanístico del Suelo de Cantabria. Modificada por la Ley } 2 / 2009 \text {, de } 3 \\
\text { de julio. } \\
\text { - Plan Regional de Ordenación del Territorio de Cantabria (en tramita- } \\
\text { ción). }\end{array}$ \\
\hline Castilla y León & $\begin{array}{l}\text { - Ley } 5 / 1999 \text {, de } 8 \text { de abril, de Urbanismo. Modificada por Ley } 10 / 2002 \text {, } \\
\text { de } 10 \text { de julio y por la Ley } 4 / 2008 \text {, de } 15 \text { de septiembre, de Medidas } \\
\text { sobre Urbanismo y Suelo. } \\
\text { - Directrices Esenciales de Ordenación del Territorio de Castilla y León, } \\
2008 \text {. }\end{array}$ \\
\hline
\end{tabular}

Fuente: Gobierno de España, Ministerio de Vivienda y elaboración propia.

Asturias y sus regiones limítrofes ofrecen, en general, un cuadro bien articulado de promotores públicos, instrumentos y tipologías de suelo industrial, con desarrollos que abarcan desde los polígonos tradicionales a los parques tecnológicos, pasando por los parques empresariales y las zonas logísticas. En lo que sigue se analizan las características de la promoción en las comunidades autónomas de Galicia, Cantabria y Castilla y León y en el apartado siguiente se profundiza en el caso asturiano, en un intento por alcanzar conclusiones válidas sobre la actividad promotora de suelo industrial. 


\subsection{La promoción de suelo industrial en Galicia}

Los diagnósticos y estudios sobre la situación del suelo industrial en Galicia coinciden en resaltar que la falta de suelo industrial ha actuado como factor de freno a la implantación de nuevos proyectos y a la ampliación de las empresas consolidadas (CONSORCIO ZONA FRANCA DE VIGO, 2003). La evolución reciente del mercado de suelo empresarial muestra dos fenómenos destacables: uno, que la demanda desborda la oferta de suelo para las empresas; y dos, que se trata de una demanda muy selectiva, con preferencia por las grandes áreas urbanas. Ambos rasgos se traducen en precios elevados tanto del suelo como del techo industrial (naves). En efecto, una de cada tres empresas que buscan dónde crecer o asentarse lo hace en los cinturones de Vigo-Pontevedra, Santiago-A Coruña y, en menor medida, Ferrol y Arousa. El litoral concentra ya un $80 \%$ de la demanda real, pero no existe suelo disponible y la escalada de precios está provocando desde 1999 una fuga de empresas, fundamentalmente hacia el norte de Portugal, donde el censo de firmas gallegas supera las 150 empresas radicadas en polígonos industriales de Oporto y Valença do Minho.

La política de suelo industrial, que recae en las consellerías de Innovación e Industria y de Vivienda y Suelo, trata de paliar la situación. Ambas instancias han puesto en marcha el Proyecto Galicia Suma 2006-2013, que prevé la creación de 2.000 hectáreas de suelo industrial (cuadro 2) con una inversión aproximada de 2.000 millones de euros. El objetivo es la promoción y creación de suelo empresarial, colaborando con universidades, cámaras de comercio e industria y entidades empresariales. Se trata, en última instancia, de impulsar la actividad económica y facilitar suelo a las empresas a un precio competitivo, con ayudas que pueden ser, en ciertos casos, a fondo perdido.

Al servicio de dicho proyecto se creó la Oficina de Promoción de Suelo Empresarial, encargada de los desarrollos de suelo, su gestión y comercialización. Desde aquí se impulsa la promoción de grandes extensiones de suelo industrial que deben actuar como polos de atracción para el inversor foráneo, independientemente del desarrollo de polígonos municipales. Las mayores bolsas de suelo se concentran en lo que se denomina "triángulo de progreso", trazado imaginario que conecta las áreas industriales del Atlántico con el interior. Una de las zonas más beneficiadas es el área metropolitana de Vigo, que concentrará unas 1.100 hectáreas, repartidas entre la plataforma logística de Salvaterra-As Neves, las previsiones del Plan General y los parques de nueva creación en Mos, Ponteareas o Redondela.

Respecto a las tipologías de intervención, se establecen cuatro clases de suelo empresarial: los parques especializados, los parques logísticos, los parques multifuncionales y los parques tecnológicos. A estos hay que sumar los parques de carácter local promovidos por los Ayuntamientos, lo que redondea la oferta pública de suelo 
industrial en la región. Sobre las formas de comercialización se imponen las modalidades de parcelas urbanizadas en venta y alquiler y de techo industrial en venta y alquiler.

Cuadro 2. Instrumentos regionales de promoción de suelo industrial.

\begin{tabular}{|l|l|c|l|}
\hline $\begin{array}{l}\text { Comunidad } \\
\text { Autónoma }\end{array}$ & Instrumento & $\begin{array}{c}\text { Periodo } \\
\text { de vigencia }\end{array}$ & $\begin{array}{l}\text { Suelo industrial } \\
\text { programado (Ha) }\end{array}$ \\
\hline Galicia & Proyecto Galicia Suma & $2006-2013$ & 2.000 \\
Cantabria & Plan Estratégico & $2008-2011$ & 1.000 \\
Castilla y León & Acuerdo Marco & $2006-2009$ & 2.000 \\
Asturias & Programa de Promoción & & \\
& de Suelo Industrial & $2005-2008$ & 580 \\
\hline
\end{tabular}

Fuente: Elaboración propia con datos de las CC.AA

\subsection{Desarrollo del suelo industrial en Cantabria}

En Cantabria destacan las pequeñas promociones oficiales de suelo industrial, mientras que escasean los grandes polígonos con una oferta de suelo competitivo, especialmente en el litoral. La implantación geográfica de los usos industriales es el gran reto del Plan de Ordenación del Litoral, que delimita Áreas Integrales Estratégicas Productivas y en ellas cualquier actuación de suelo industrial debe desarrollarse mediante un Proyecto Singular de Interés Regional (PSIR).

Los PSIR son instrumentos de planeamiento territorial que tienen por objeto regular la implantación de instalaciones industriales, grandes equipamientos y servicios de especial importancia con asiento en más de un término municipal o que trasciendan dicho ámbito por su incidencia económica. En la tramitación intervienen la Comisión Regional de Ordenación del Territorio (otorga la aprobación inicial) y el Gobierno de Cantabria (aprobación definitiva). No obstante, como requisito previo a la tramitación, está la necesidad de la declaración de interés regional, que corresponde al Gobierno de Cantabria a propuesta del consejero competente en materia de ordenación territorial, previa audiencia de los Ayuntamientos afectados.

La Sociedad de Suelo Industrial de Cantabria (SICAN) es el principal instrumento para el desarrollo de la política de suelo industrial. Está participada mayoritariamente por el Gobierno autonómico, además de Caja Cantabria, Sodercan y la Cámara de Comercio, Industria y Navegación Cántabra. A través de SICAN se impulsa la constitución de una red de polígonos industriales distribuidos de manera más o menos 
homogénea en torno a los principales ejes de transportes y comunicaciones de la región, factor que eleva las posibilidades de implantación de todo tipo de industrias.

En el plano instrumental destaca el Plan Estratégico 2008-2011, que propone desarrollar unas 1.000 hectáreas de suelo industrial en 19 polígonos industriales (cuadro 2). También se incluyen en este Plan reservas de suelo para actividades económicas en general. Las tipologías de intervención previstas son: polígonos industriales, parques empresariales, parques científico-tecnológicos y las llamadas Áreas Industriales Estratégicas Productivas. La comercialización es responsabilidad de SICAN, que opta por la fórmula de la venta de la parcela urbanizada, aunque también se contempla la posibilidad del alquiler. En relación con el mantenimiento y gestión de los polígonos y áreas empresariales consolidadas, se impulsa la figura de las Entidades de Conservación, una modalidad de gestión que progresivamente se está implantando en todos los polígonos y parques empresariales.

\subsection{Castilla y León: suelo industrial sin programación integrada}

En Castilla y León el suelo industrial se reparte entre algo más de 300 polígonos y parques empresariales de las nueve provincias de la Comunidad. En línea con lo observado en otras CC.AA., la política de suelo industrial aspira a superar la tipología tradicional de polígonos al uso y pone el acento en promover parques empresariales que ofrezcan un perfil de servicios más avanzados, en respuesta a la demanda empresarial y en sintonía con los objetivos de innovación industrial y sus espacios por excelencia: los parques tecnológicos y científicos (GONZÁLEZ y BENITO, 2008; SANTOS y PEIRET, 2001).

La gestión y ejecución de actuaciones de promoción y de obtención de suelo industrial fue competencia hasta fecha reciente de Gesturcal, entidad adscrita a la Consejería de Industria, Comercio y Turismo. Pero en septiembre de 2008 se produjo la fusión con la empresa pública Parques Tecnológicos de Castilla y León, dando lugar a una nueva estructura organizativa con cuatro direcciones generales (parques, infraestructuras y obras, proyectos y estudios, y planificación). El objetivo de esta reorganización es dotar a la entidad resultante, ADE Parques Tecnológicos de Castilla y León, de mayor eficiencia y agilidad para poner a disposición de los empresarios unas 2.000 hectáreas de suelo empresarial.

A diferencia de las regiones vecinas, la Junta de Castilla y León carece de un programa de suelo industrial integrado, aunque existió el llamado Plan Regional Sectorial de Espacios Industriales y de Actividad Económica 2000-2006 que no tuvo aplicación. En efecto, en el año 2000 la Junta planteó potenciar la disponibilidad de suelo indus- 
trial a precios favorables y con infraestructuras adecuadas en todas las provincias a través de un documento integrador que después no se gestionó como tal, ni las actuaciones previstas se concretaron en sus términos exactos. Es más, los polígonos ejecutados en ese período no se consideraron la expresión de un plan oficial, sino intervenciones estratégicas puntuales.

En lugar de un verdadero plan de suelo la Junta ha ejecutado el Acuerdo Marco para la Competitividad e Innovación Industrial 2006-2009, que propone generar 1.000 hectáreas de suelo industrial (cuadro 2) y contiene tres ejes de actuación con referencia explícita a la localización y el suelo industrial:

- Eje de dimensión territorial y sectorial: plantea el objetivo de mejorar la cohesión y el equilibrio territorial en el desarrollo industrial de Castilla y León a través, entre otras actuaciones, de una oferta de suelo industrial que aproveche las oportunidades de los entornos más dinámicos (Madrid y País Vasco) y con micro-polígonos rurales ligados a la actividad empresarial del medio.

- Eje de infraestructuras de apoyo a la industria: propone garantizar la disponibilidad de suelo y naves que den soporte físico a la actividad industrial, así como el acceso a las fuentes de energía, a los servicios de telecomunicaciones y a los servicios de gestión medioambiental.

- Eje de anclaje territorial de las empresas: contempla medidas orientadas a fomentar el asentamiento de las empresas en Castilla y León, su vinculación al territorio y el control del impacto negativo de los procesos de reestructuración industrial y deslocalización de empresas.

Las tipologías de intervención incluyen polígonos industriales, parques empresariales y parques tecnológicos. Como antes se ha apuntado, en las zonas rurales se promueven micropolígonos con el fin de aprovechar las sinergias del entorno, lo que permite fijar población y generar actividad económica. Entre las formas de comercialización también aquí destacan la venta de parcelas urbanizadas y el alquiler de naves o techo industrial (MATÍNEZ y LUENGO, 2005; RAMA y CALATRAVA, 2002).

\section{La experiencia planificadora en Asturias: actuaciones y propuestas}

En Asturias la mayoría de los asentamientos industriales y áreas empresariales son el resultado de un proceso de lenta transformación del suelo de la Zona Central, la más urbanizada y mejor comunicada, para usos económicos. El Principado de Asturias, a través de su agencia de desarrollo regional, IDEPA, es el principal agente promotor y gestor del suelo para las empresas, actuando según criterios que obedecen a la necesidad de asegurar la dotación de suelo requerida en cada momento para 
apoyar la consolidación y expansión del tejido de pymes y grandes empresas, toda vez que la iniciativa privada ha dado muestras de un escaso interés por liderar el proceso de creación de suelo industrial, un negocio con limitadas expectativas de ganancia y alto riesgo, sobre todo en etapas de crisis pasadas (reconversión industrial y ajustes productivos en los años de 1980 y 1990) y en momentos de contracción general de la inversión y los negocios como el actual (BENITO, 2006; APIA, 2004).

\subsection{Antecedentes de la promoción oficial de polígonos industriales en Asturias}

En Asturias, la disponibilidad de suelo apto para las empresas ha sido y sigue siendo limitada debido a su accidentado relieve, un factor geográfico insoslayable. Además, acondicionar terrenos industriales exige hacer frente a una estructura de la propiedad muy atomizada, a importantes condicionantes medioambientales (protección del litoral, espacios de interés paisajístico, reservas naturales), a una fuerte competencia de los usos residenciales, a carencias en infraestructuras básicas (agua, gas, suministro eléctrico) y, en fin, a una tramitación urbanística compleja que alarga los plazos e impide atender con puntualidad la demanda. En estas condiciones la iniciativa privada se ha mostrado, en general, reacia a intervenir en el mercado del suelo industrial, así que los agentes públicos han debido llevar la iniciativa en la creación de suelo para empresas.

Otro rasgo del suelo industrial en Asturias es su fuerte concentración: la oferta en todas las modalidades registradas, desde polígonos y parques empresariales hasta parques tecnológicos, centros de empresas, incubadoras, etcétera, tiende a localizarse en el Área Central, apoyada en el soporte urbanístico del llamado "Ocho Asturiano" del que forman parte las ciudades de Oviedo, Gijón, Avilés, Mieres y Langreo. En este reducido espacio metropolitano se concentran las principales infraestructuras de transporte y comunicaciones y los recursos de todo tipo, por lo que la demanda presiona con intensidad creciente en el área. Lo hizo en el pasado, en los años del Polo de Desarrollo y de auge de la industria pesada, y lo hace en el presente, sobre la base de una industria ligera que cobra importancia a impulso de las pymes. Pero el suelo es un recurso limitado y cada día son más los usos que compiten por su consumo, a tenor de la urbanización creciente del territorio.

El resultado de estos y otros factores es un déficit crónico de la oferta de suelo industrial, muy selectiva en términos espaciales y con un claro protagonismo de los promotores públicos que, como no puede ser de otro modo, ligan los objetivos de las políticas económica e industrial a las intervenciones en materia de suelo empresarial.

Con todo, una mirada al pasado y un análisis detenido del presente pone de manifiesto que desde mediados de los años de 1960 hasta la actualidad el volumen de 
suelo industrial consolidado y en oferta en Asturias ha crecido en todas las comarcas y ámbitos territoriales: desde el Área Central y las comarcas mineras, hasta las zonas Oriental y Occidental. Una expansión que podría haber sido mayor de no mediar la larga crisis industrial de los años de 1980 y 90, y si las políticas de reindustrialización hubiesen tenido mayor calado en sus objetivos y mejores resultados finales.

Desde los primeros polígonos industriales que se planifican en los años 60, dentro de la política de polos de desarrollo (como Silvota en Llanera y Vega de Arriba en Mieres), la oferta en Asturias aparece supeditada a las peculiaridades del tejido empresarial: primero caracterizado por el peso de las grandes empresas públicas y su especialización en actividades básicas; y después, significado por la escasez de pymes y la limitada diversificación sectorial. A esto hay que añadir unos precios relativamente altos que no siempre se corresponderán con el nivel de calidad de los polígonos, muchos de ellos con notables deficiencias en accesos, servicios y equipamientos arrastradas durante años.

No obstante, el precio nunca fue el principal freno para la ocupación real de los polígonos. La demanda siempre valoró por delante del precio otros factores como la localización de los terrenos, su accesibilidad y su nivel de urbanización, servicios y dotaciones. Un ejemplo: Asipo, de promoción privada y uno de los polígonos más caros en su momento, fue un éxito desde su puesta en marcha en 1976 porque sus condiciones de infraestructura, gestión y servicios tenían el nivel que cabía esperar de un polígono moderno. Ahora bien, la escasez de suelo en esos años también permite explicar que otros polígonos privados, como Bankunión I y Bankunión II en Gijón, deficitarios en servicios y con una infraestructura precaria, tuvieran éxito de ventas y ocupación.

Un caso singular lo representa Silvota, el primer macropolígono de Asturias, proyectado en 1964 por la Gerencia de Urbanismo (luego INUR y SEPES) con casi 600 hectáreas de superficie. Pues bien, de este gran polígono sólo se ejecutará la primera fase, llamada Silvota I y con 139 hectáreas de superficie (figura 1) debido a los problemas de gestión y las graves deficiencias en infraestructuras y servicios, que hicieron fracasar la venta de parcelas y llevaron a SEPES, heredero de la promoción, a abandonar el resto del proyecto en los años 80.

Las más de 400 hectáreas de suelo industrial no desarrollado de Silvota pasarán con los años a tener otros propietarios, entre ellos Mercasturias y el Principado, que localizarán en estos terrenos un gran mercado mayorista de frutas y verduras y el Parque Tecnológico de Llanera, respectivamente. En concreto el parque tecnológico se puso en marcha en 1991 siguiendo la tendencia en toda España a producir suelo para las empresas de alta tecnología, un modelo que Europa copia, con resultados muy desiguales, del Silicon Valley californiano. Usos terciarios e industriales de nuevo 
cuño para un viejo proyecto de desarrollo industrial en el mejor emplazamiento de la región, como lo demuestra su posterior colmatación y alto grado de aceptación por las empresas, si bien los proyectos industriales tardaron tiempo en arraigar debido a la crisis industrial y la reconversión sectorial, que no se cierra hasta bien avanzada la década de 1990.

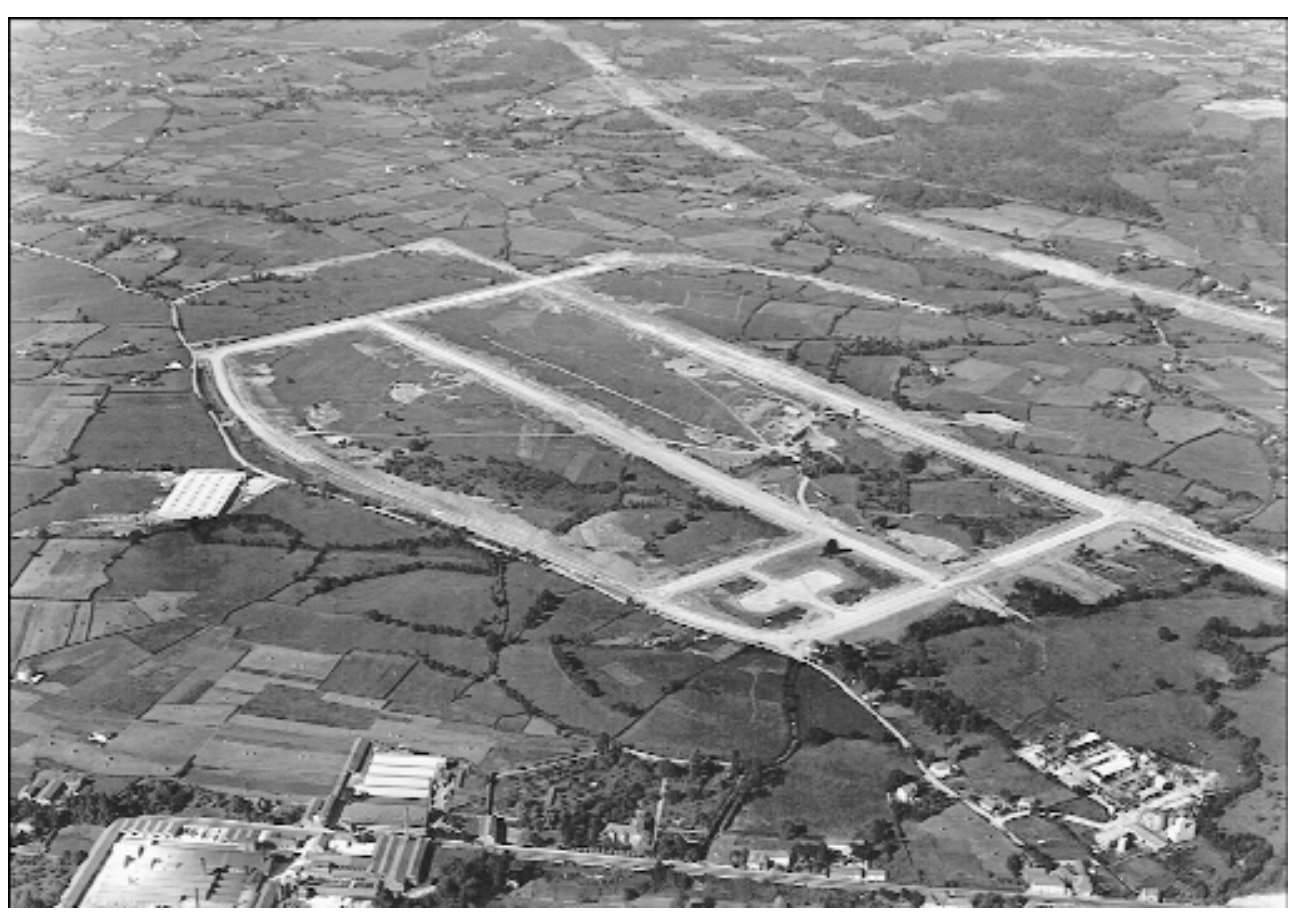

Figura 1. Delimitación del polígono de Silvota, una promoción del INUR en los años de 1960 en el marco del Polo de Desarrollo de Oviedo. Fuente: Archivo particular.

Por su parte, las políticas de reindustrialización de mediados de los años de 1980 van a suponer un crecimiento de la oferta de suelo, ya que al amparo de las ZUR (Zonas de Urgente Reindustrialización), se crean cuatro polígonos industriales: Fábrica, en Mieres; Riaño I en Langreo, Mora-Garay en Gijón y Las Arobias en la Ría de Avilés, construidos entre 1986 y 1988. En total 70 hectáreas brutas de suelo industrial que se suman a la oferta heredada de las promociones oficiales de la década anterior: las 139 hectáreas de Silvota y las 12,5 hectáreas del polígomo Vega de Arriba de Mieres. 
En estos mismos años va tomando cuerpo el corredor industrial de Siero, escenario de una amalgama de asentamientos industriales espontáneos, polígonos planificados, empresas aisladas y una prematura oferta de naves en la zona de Granda. El proceso que explica la formación de este espacio industrial no es otro que la expulsión de la industria urbana hacia áreas periféricas, donde las empresas encuentran suelo abundante, más barato que en la ciudad y sin apenas restricciones de uso. A este fenómeno se suma el desarrollo de una industria alejada de los focos tradicionales (Avilés, Gijón, Mieres y Langreo), muy orientada al mercado de consumo y supeditada a las infraestructuras de transporte rápido, que toma como referencia para su localización la proximidad de Oviedo, reforzado en su función de centro de distribución y servicios.

\subsection{La importancia de los promotores públicos de suelo empresarial}

El abanico de agentes públicos que intervienen en la producción de suelo industrial se abre a lo largo de los 90 y principios de 2000. A Sepes, los Ayuntamientos y el Gobierno del Principado se unen Sogepsa, Sepi-Infoinvest y el IDEPA. Los recursos públicos para suelo industrial se nutren, asimismo, de los fondos mineros aportados por el Ministerio de Economía, a través de sucesivos planes que contemplan crear polígonos industriales en los municipios más afectados por la reestructuración del carbón, con el fin de propiciar un desarrollo industrial alternativo y crear empleo.

Llegados a este punto hay que destacar los planes impulsados por el Gobierno regional y ejecutados por el IFR primero y después por el IDEPA. Estos planes oficiales abarcan los períodos de 1990-1993, 2001-2004 y 2005-2008 y tienen como objetivo primordial impulsar la expansión de la oferta de suelo para favorecer el desarrollo empresarial y captar inversiones. El plan de 1990 proponía crear 525 Ha brutas de suelo repartido en 22 polígonos; el plan de 2001 propuso 651 Ha para 27 polígonos y parques empresariales, y el plan 2005-2008 planteaba desarrollar 580 Ha en 38 parques empresariales. Una aportación de suelo ciertamente importante (sobre todo si se tiene en cuenta el alto nivel de cumplimiento de estos planes) que, sin embargo, no ha permitido equilibrar la oferta y la demanda. El último plan aprobado para el periodo 2009-2012 asume el modelo territorial del precedente y reitera algunas localizaciones y desarrollos heredados del anterior (figura 2).

Además de crear nuevo suelo, los planes regionales incluyen acciones de mejora de la calidad de los polígonos, un aspecto que preocupa cada vez más por su relación no solo con el medio ambiente, sino también con la eficiencia productiva y la propia dinámica empresarial. Destaca la influencia ejercida por las asociaciones de empresarios y las organizaciones de polígonos, APIA y CEPE entre ellas, que insisten en la mejora continuada de los polígonos y en la calidad del nuevo suelo industrial. 


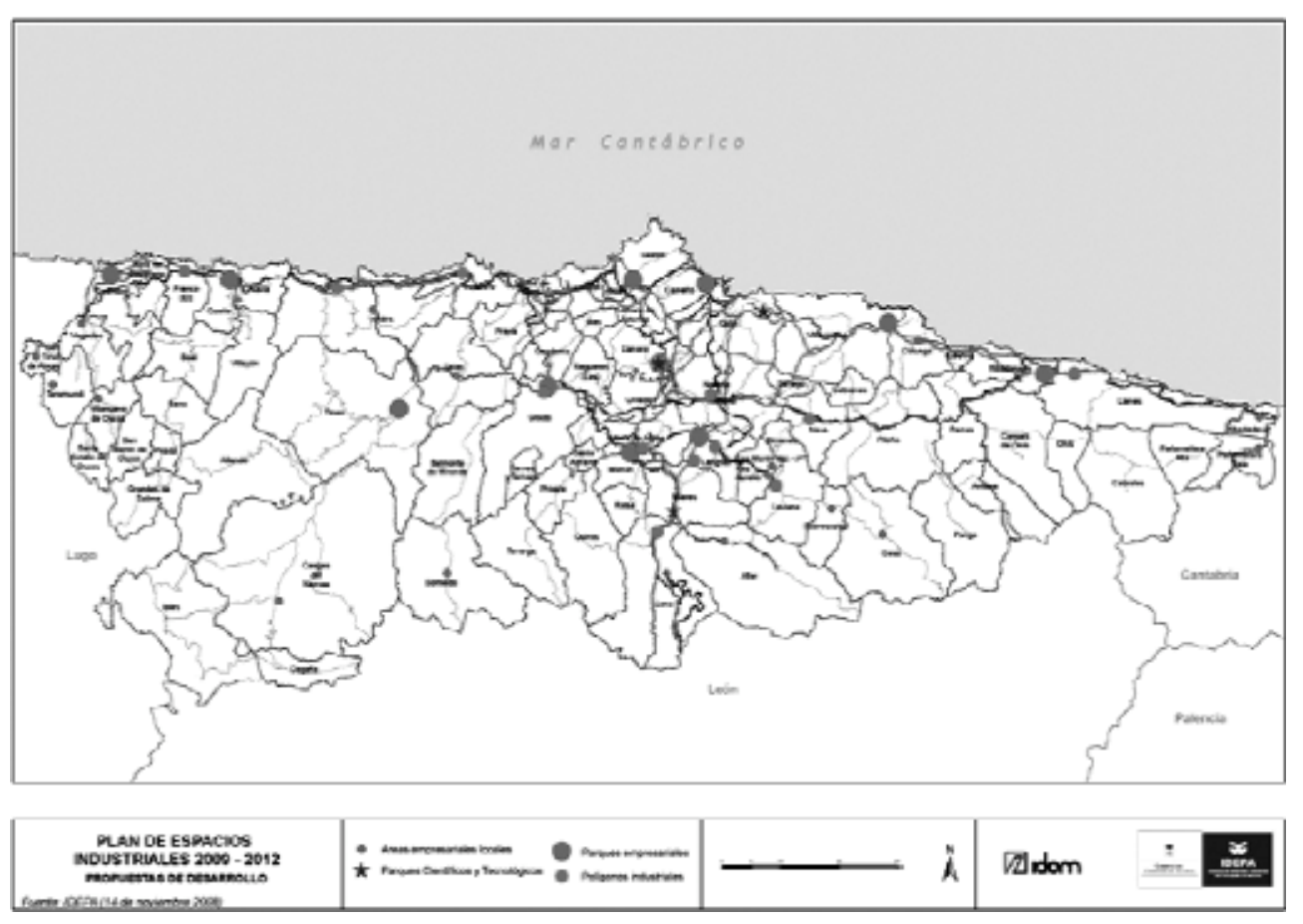

Figura 2. Propuesta de suelo industrial para el periodo 2009-2012 en Asturias. Fuente: IDEPA.

En otro orden, se constata que los planes de suelo del Principado han evolucionado hacia un mayor compromiso con el equilibrio regional y la ordenación del territorio. En tal sentido, los planes arriba indicados proponen nuevos desarrollos de suelo industrial en localizaciones estratégicas del Área Central, pero también en las comarcas y subcomarcas funcionales y en las zonas rurales de la región, un modelo territorial que refuerza la primacía del espacio metropolitano central y que se aleja de propuestas extremas como son crear grandes áreas empresariales en localizaciones muy concretas y selectivas, con un impacto enorme y de difícil gestión; o bien atomizar la oferta asignando suelo a todos los municipios, se justifique o no, lo cual no deja de ser, cuando menos, un despilfarro ya que el proceso de difusión industrial tiene su propia lógica y la mera oferta de suelo no es garantía de fijación de inversiones (BIELZA, 2008; ROMERO y FARINÓS, 2004; GARRIDO, 2007). 


\subsection{Impulso institucional y concertado a la creación de suelo industrial: el ACEBA}

El Acuerdo para la Competitividad, el Empleo y el Bienestar de Asturias 2008-2011 (ACEBA) firmado entre el Principado de Asturias, la Federación de Empresarios (FADE) y los sindicatos UGT y Comisiones Obreras, reedita la concertación regional y constituye actualmente el principal marco de referencia de la política asturiana. Contempla un total de 1.372 millones de euros destinados a promoción económica, generación de suelo industrial, impulso a la internacionalización de la empresa asturiana, fomento de emprendedores, $\mathrm{I}+\mathrm{D}+\mathrm{i}$, desarrollo de la sociedad de la información, prevención de riesgos y salud laboral, vivienda y cohesión social. La inversión pública prevista alcanza los 2.319 millones de euros y se destinará al desarrollo de infraestructuras de comunicación, saneamiento, abastecimiento, infraestructuras ligadas al medioambiente, a la prestación de servicios públicos al ciudadano y para el desarrollo y urbanización de una gran zona logística y para viviendas (GOBIERNO DEL PRINCIPADO DE ASTURIAS, 2008).

El documento dedica un apartado completo al suelo industrial, con una introducción que confirma que, a pesar de los avances logrados por los dos programas oficiales de suelo industrial puestos en marcha en los últimos años, la dotación de este recurso en Asturias continúa siendo escasa. En consecuencia, el Acuerdo plantea el compromiso de elaborar un nuevo Programa de Suelo Industrial, 2009-2012 cediendo la responsabilidad de su desarrollo y gestión al IDEPA, en línea con los planteamientos expuestos en el apartado anterior.

El ACEBA propone desarrollar suelo industrial con preferencia en el Área Central de Asturias debido a la consolidación aquí de importantes asentamientos industriales, con una demostrada dinámica expansiva y fuerte capacidad de atracción (entre los que destacan los polígonos de Asipo y Silvota, los parques tecnológicos de Llanera y Gijón, el parque empresarial PEPA de Avilés o ciertas asentamientos industriales de Siero) y a las preferencias expresadas por la demanda, que apuntan a los municipios de Gijón, Llanera, Siero, Oviedo y Avilés-Carreño. El objetivo es crear más de 40 polígonos industriales que representan 10,4 millones de metros cuadrados brutos de suelo industrial (cuadro 3) con un presupuesto de 48 millones de $€$ También se fija como objetivo adicional la previsión de suelo a 10 años, a fin de avanzar en la compleja tramitación urbanística e ir acortando plazos para la obtención definitiva de parcelas industriales.

Además, se plantea el desarrollo de 420 hectáreas del proyecto ZALIA, Zona de Actividades Logísticas e Industriales de Asturias. Se incluyen criterios cualitativos en la localización del suelo industrial: precio competitivo y con las mejores dotaciones posibles (accesos, suministros energéticos, acceso a banda ancha y adecuadas condi- 
Cuadro 3. Propuesta del ACEBA de nuevo suelo industrial para Asturias.

\begin{tabular}{|c|c|c|c|}
\hline $\begin{array}{l}\text { Poligono industriall } \\
\text { Parque empresarial }\end{array}$ & Municipio & $\begin{array}{l}\text { Superficie } \\
\text { bruta }\left(m^{2}\right)\end{array}$ & $\begin{array}{l}\text { Superficie } \\
\text { neta }\left(m^{2}\right)\end{array}$ \\
\hline Lloreda A & Gijón & 984.519 & 578.771 \\
\hline La Granda II & Carreño & 315.901 & 191.246 \\
\hline Bobes - San Miguel & Siero & 1.110 .534 & 665.131 \\
\hline La Cardosa & Grado & 84.778 & 48.000 \\
\hline Guadamía ampliación & Ribadesella & 133.100 & 56.954 \\
\hline Barres III & Castropol & 167.000 & 95.139 \\
\hline Llaviada & Boal & 44.011 & 26.015 \\
\hline El Couso & Grandas de Salime & 20.099 & 12.936 \\
\hline Trevías & Valdés & 57.478 & 33.138 \\
\hline $\begin{array}{c}\text { Parque Artesanal } \\
\text { de Taramundi } \\
\text { Ampliación Parque }\end{array}$ & Taramundi & 51.675 & 36.061 \\
\hline Tecnológico de Asturias & Llanera & 74.401 & 44.641 \\
\hline Ampliación Silvota & Llanera & 315.133 & 166.632 \\
\hline Ampliación Asipo & Llanera & 385.775 & 270.042 \\
\hline Recta de Lledo III & Piloña & 27.311 & 17.333 \\
\hline Parque de la Sidra & Nava & 82.475 & 40.438 \\
\hline Santianes & Sariego & 132.100 & 90.706 \\
\hline Piñeres & Llanes & 88.965 & 48.338 \\
\hline Río Pinto III & Coaña & 89.885 & 52.093 \\
\hline Novales & El Franco & 49.860 & 28.185 \\
\hline Puente de Bobia & Somiedo & 15.200 & 9.120 \\
\hline Lloreda B & Gijón & 117.127 & 59.719 \\
\hline Valliniello - Retumés & Avilés & 550.000 & 260.800 \\
\hline La Rasa & El Franco & 164.034 & 11.375 \\
\hline Pilando & El Franco & 72.610 & 52.230 \\
\hline Almuña III & Valdés & 44.015 & 34.075 \\
\hline Aeropuerto & Castrillón & 688.656 & 497.868 \\
\hline Santianes III & Sariego & 118.876 & 70.048 \\
\hline Santianes Lamasanti & Sariego & 165.730 & 127.002 \\
\hline La Gasolinera & Somiedo & 9.326 & 5.873 \\
\hline Guadamía 2 & Ribadesella & 226.816 & 165.288 \\
\hline Sevares & Piloña & 61.259 & 39.918 \\
\hline El Taquín & Colunga & 78.098 & 52.539 \\
\hline Piñeres 2 & Llanes & 263.553 & 147.326 \\
\hline Posada 3 & Llanes & 49.672 & 27.500 \\
\hline San Roque 2 & Llanes & 125.037 & 75.093 \\
\hline Parque de la madera & Valdés & 202.108 & 151.581 \\
\hline La Garganta & Villanueva de Oscos & 34.994 & 9.408 \\
\hline La Rasa Luces - Selorio & Colunga-Villaviciosa & 550.000 & 255.000 \\
\hline La Junquera - San Esteban & Muros de Nalón & 56.641 & 38.233 \\
\hline Oviñana & Cudillero & 54.371 & 32.623 \\
\hline
\end{tabular}




\begin{tabular}{|c|c|c|c|}
\hline $\begin{array}{c}\text { Polígono industrial/ } \\
\text { Parque empresarial }\end{array}$ & Municipio & $\begin{array}{c}\text { Superficie } \\
\text { bruta }\left(\mathrm{m}^{2}\right)\end{array}$ & $\begin{array}{c}\text { Superficie } \\
\text { neta }\left(\mathrm{m}^{2}\right)\end{array}$ \\
\hline La Cobertoria & Lena & 70.211 & 31.133 \\
Río Pinto IV & Coaña & 300.000 & 180.000 \\
Grado Este & Grado & 900.000 & 585.000 \\
Ampliación Asipo & Llanera & 805.341 & 483.204 \\
Ampliación Silvota & Llanera & 515.987 & 309.592 \\
\hline \multicolumn{2}{|r|}{ TOTAL } & $\mathbf{1 0 . 4 5 4 . 6 6 2}$ & $\mathbf{6 . 2 1 3 . 3 4 7}$ \\
\hline
\end{tabular}

Fuente: Elaboración propia con datos del ACEBA y el IDEPA, 2008.

ciones medioambientales). También una línea de suelo industrial dirigido a emprendedores. Se trata de proponer una oferta atractiva a los colectivos de pequeñas industrias y jóvenes empresarios a fin de mejorar la competitividad de la región como área de atracción de emprendedores. Asimismo, se proponen medidas orientadas a potenciar la actividad económica, tales como la promoción de naves industriales en zonas de mayor demanda y con especial atención a emprendedores.

\subsection{Perspectivas en el desarrollo de áreas empresariales}

Iniciada la década de 2000 el conjunto de los agentes económicos asume que las actividades productivas dependen cada vez más de factores ligados a la eficiencia de los servicios, las infraestructuras avanzadas y la imagen que el propio espacio industrial es capaz de exhibir y proyectar hacia el exterior.

En relación con esto, en los últimos años se afianza la oferta de suelo tecnológico y científico y en los parques de Llanera y Gijón. También la oferta de techo para actividades TIC encuentra un hueco en los parques tecnológicos, dotados con centros de empresas y otras promociones.

A pesar de esta infraestructura, en Asturias el tejido empresarial ligado a las nuevas tecnologías sigue siendo débil, quizá por su tardío desarrollo. Una conclusión sugerida por recientes trabajos es que los parques tecnológicos asturianos apenas han contribuido a reforzar el sistema ciencia-tecnología-empresa, al margen de que se trate de áreas empresariales que, sin duda, mejoran el paisaje industrial y fortalecen la imagen de modernidad del tejido productivo asturiano (ONDATEGUI y SÁNCHEZ) 2004). Pero esto no es suficiente en un escenario de economía globalizada que apuesta por la innovación y el conocimiento como elementos clave del sistema productivo. En un contexto de expansión de las redes globales de innovación y de mer- 


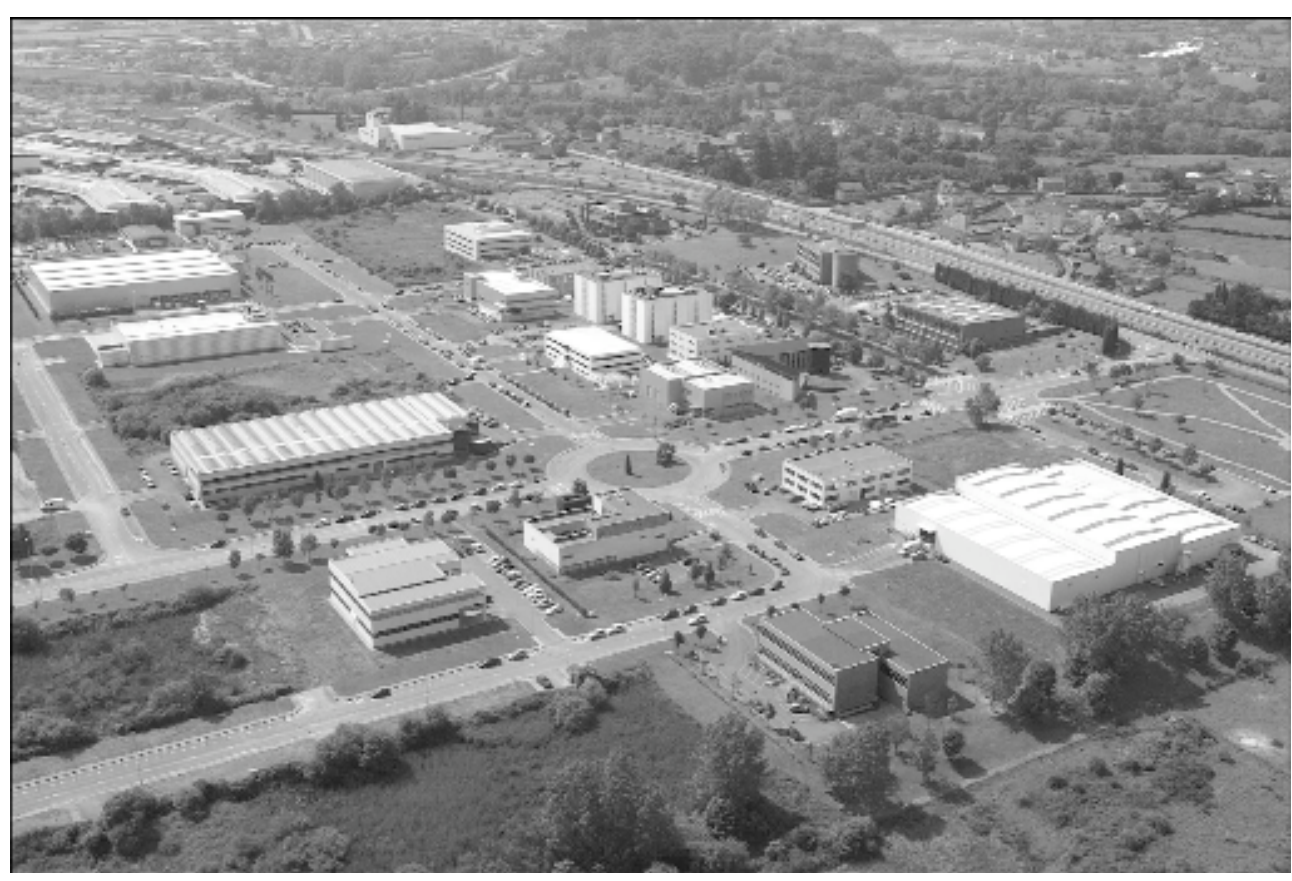

Figura 3. Vista general del Parque Tecnológico de Llanera en 2008.

Fuente: Archivo de los autores.

cados, los parques y áreas empresariales deberían cumplir la función de reunir y concentrar el conocimiento, facilitando un entorno que hace posible, al decir de los expertos, la creatividad, la transferencia y el crecimiento de empresas y negocios (APTE, 2003).

\section{Conclusiones}

El suelo industrial aparece como un factor de desarrollo regional que los Gobiernos autonómicos potencian con instrumentos que crean nuevo suelo, mejoran la calidad del existente y propician localizaciones a las empresas, facilitando las nuevas inversiones, las ampliaciones y la relocalización de actividades, más allá de las áreas saturadas o inconvenientes. Además de una vertiente sectorial, tienen una dimensión territorial y fijan la extensión de suelo a producir y su emplazamiento. Por 
lo general, refuerzan las tendencias de localización heredadas o generadas por procesos espontáneos, si bien plantean actuaciones que atajan la dispersión indiscriminada y favorecen la concentración allí donde el territorio ofrece mejores soportes a la producción. Los municipios urbanos y metropolitanos son los que acaparan el mayor volumen de suelo industrial, pero también se favorecen dinámicas de industrialización en espacios rurales con demanda de suelo empresarial.

Por su parte, la ordenación de los espacios industriales sigue pautas comunes, bien a través de las directrices correspondientes, bien por medio de la legislación urbanística, con unas indicaciones específicas y precisas, lo que en principio es una garantía de crecimiento territorial equilibrado y coherente. Los aspectos medioambientales también se salvaguardan en términos normativos, pero la experiencia demuestra que la práctica urbanística y la empresarial, cada una por su lado, tienden a reducirlos a su mínima expresión.

Pese a los empeños oficiales, la planificación de suelo industrial y la promoción pública no responden a las exigencias del mercado: por lo general la oferta está por debajo de la demanda, lo que se agrava por el hecho constatado de que el desarrollo urbanístico del suelo industrial conlleva una tramitación lenta y compleja (ritmo lento), lo que hace que las parcelas se pongan a la venta cuando las necesidades (ritmo rápido) se han multiplicado; además, la demanda presiona selectivamente, y no todo el suelo se genera donde más se reclama. En consecuencia: ¿debe potenciarse la promoción privada de suelo industrial como complemento de la pública? El riesgo puede ser un crecimiento menos ordenado y más especulativo del suelo industrial y, por ende, de las áreas empresariales. El desafío: conciliar el crecimiento satisfactorio (y suficiente) de los espacios económicos y la ordenación y gestión sostenible del territorio.

\section{Bibliografía}

APIA (2004). Estudio de los polígonos industriales en Asturias. Gijón (soporte CD).

APTE (2003). Los parques científicos y tecnológicos: una contribución fundamental al sistema de ciencia y tecnología en España. Málaga, 208 p.

Benito del Pozo, P. (2006). "Promoción y valorización del suelo industrial en Asturias". Boletín de la Asociación de Geógrafos Españoles, 42, p. 99-120.
Benito del Pozo, P.; López, A.; González, N. (2006). Suelo industrial y territorio en León. León, Secretariado de Publicaciones de la Universidad de León.

Bielza de Ory, V. (2008). Introducción a la ordenación del territorio. Un enfoque geográfico. Zaragoza, Servicio de Publicaciones de la Universidad de Zaragoza.

COMISIÓN EUROPEA (1999). Estrategia Territorial Europea. Hacia un desarrollo 
equilibrado y sostenible del territorio de la UE. Luxemburgo, Comunidades Europeas.

COMISIÓN EUROPEA (2007). Agenda Territorial de la Unión Europea. Luxemburgo. Comunidades Europeas.

CONSEJERÍA DE MEDIO AMBIENTE Y URBANISMO (1992). Directrices Regionales de Ordenación del Territorio. Oviedo, Servicio de Publicaciones del Principado de Asturias.

CONSORCIO ZONA FRANCA DE VIGO (2003). Parques empresariales de Galicia e Norte de Portugal. Vigo.

Gago, J.; García, J.M. (dirs.) (1997). Ordenación de áreas industriales. Valladolid, Junta de Castilla y León.

Garrido, R. (2007). Localización y movilidad de empresas en España. Fundación EOI, Madrid.

GOBIERNO DEL PRINCIPADO DE ASTURIAS (2008). ACEBA. Acuerdo para la Competitividad, el Empleo y el Bienestar de Asturias, 2008-2011. Oviedo, 373 p.

González, N.; Benito, P. (2008). "Política industrial y suelo industrial: especial referencia a Castilla y León". Pecunia, 7, 101123.

Martínez, L. C.; Luengo, J.A. (2005) "Localización de la actividad manufacturera en Castilla y León. Los dinamismos espaciales inducidos por la industria".
Ería. Revista de Geografía, 67, pp. 155172

Mémdez, R. (2006). "Políticas de promoción y ordenación industrial en las áreas urbanas", en Mémdez, R.; Pascual, H.: Industria y ciudad en España: nuevas realidades, nuevos retos. Madrid, ThomsonCivitas, pp. 51-74.

Ondátegui, J. C.; Sánchez, J. L. (2004). "Parques científico-tecnológicos en España: de la planificación a la evaluación”. Anales de Geografía de la Universidad Complutense, 24, pp. 31-51

Pujadas, R.; Font, J. (1998). Ordenación y planificación territorial. Madrid, Síntesis.

Rama, R.; Calatrava, A. (2002). "Nuevos procesos de industrialización rural en España”, en Gómez, C. y González, J.J.: Agricultura y sociedad en el cambio de siglo. Madrid, Mac Graw-Hill, pp. 477-503.

Romero, J.; Farinós, J. (eds.) (2004). Ordenación del territorio y desarrollo territorial. Gijón, Trea.

Santos, L.; Peiret, A. (2001). "Articulación regional y comarcas en Castilla y León: las Directrices de Ordenación del Territorio". $B A G E$, 32, pp. 177-190.

Sanz, J. I. (1982). "Algunas notas sobre la planificación de suelo industrial: la actividad industrial como una actividad más". Ciudad y Territorio, 54, pp.15-18. 